MATHEMATICS OF COMPUTATION

Volume 68, Number 225, January 1999, Pages 145-168

S 0025-5718(99)01028-5

\title{
ABSORBING BOUNDARY CONDITIONS FOR ELECTROMAGNETIC WAVE PROPAGATION
}

\author{
XIAOBING FENG
}

\begin{abstract}
In this paper, the theoretical perfectly absorbing boundary condition on the boundary of a half-space domain is developed for the Maxwell system by considering the system as a whole instead of considering each component of the electromagnetic fields individually. This boundary condition allows any wave motion generated within the domain to pass through the boundary of the domain without generating any reflections back into the interior. By approximating this theoretical boundary condition a class of local absorbing boundary conditions for the Maxwell system can be constructed. Well-posedness in the sense of Kreiss of the Maxwell system with each of these local absorbing boundary conditions is established, and the reflection coefficients are computed as a plane wave strikes the artificial boundary. Numerical experiments are also provided to show the performance of these local absorbing boundary conditions
\end{abstract}

\section{$\S 1$. INTRODUCTION}

Electromagnetic phenomena in a perfect medium [3] are described with the help of two vector fields $\vec{E}$ and $\vec{H}$, the electric field and the magnetic field. The properties of the medium in which the waves propagate are characterized by the two material constants $\varepsilon$ and $\mu$, the permittivity and the permeability of the medium. The forces which generate the waves are described as charge density and current density, which we denote by $\rho$ and $\vec{J}$.

The relations between vector fields $\vec{E}, \vec{H}$ and the material constants $\varepsilon, \mu$ are formulated by the following Maxwell system:

$$
\begin{gathered}
\operatorname{div} \vec{E}=\frac{\rho}{\varepsilon}, \\
\operatorname{div} \vec{H}=0, \\
\frac{\partial \vec{E}}{\partial t}=\frac{1}{\varepsilon}(\operatorname{curl} \vec{H}-\vec{J}), \\
\frac{\partial \vec{H}}{\partial t}=-\frac{1}{\mu} \operatorname{curl} \vec{E} .
\end{gathered}
$$

To make this Maxwell system solvable, we have to prescribe the initial condition and the boundary condition if the system is not considered in the entire space $\mathbb{R}^{3}$. In this paper we will consider it in a half-space domain.

Received by the editor January 11, 1994 and, in revised form, June 27, 1994 and May 24, 1997.

1991 Mathematics Subject Classification. Primary 65M99; Secondary 35L50.

Key words and phrases. Maxwell system, absorbing boundary conditions, Laplace-Fourier transform, Kreiss criterion. 
There are many important problems of numerical simulation of waves whose significant components propagate through an infinite domain. Typical examples are found in underwater acoustics, seismic wave propagations, and the electromagnetic wave scattering related to antennas. Because of the limitations of both speed and memory of present day computers, one must solve these problems in a finite domain. A natural way to do so is to introduce artificial computational boundaries without changing the governing differential equations. On these artificial boundaries one need to impose some boundary conditions so that waves can be transmitted through these boundaries.

Theoretically, pseudo-differential operators can be constructed on the artificial boundaries for perfect transmission of outgoing waves. Unfortunately, except in the most trivial one-dimensional cases, these boundary conditions are non-local both in space and time and are not practical for numerical approximations. Therefore, one must essentially approximate in a judicious fashion the perfect absorbing boundary pseudo-differential operators and get a class of computationally rigorous boundary conditions of differential operators of order $N$, which are called " $N$ th order absorbing boundary conditions". With these absorbing boundary conditions, which are local in space and time, there arise two problems to be studied: the well-posedness of the original problems in an artificial domain and reflection of waves at artificial boundaries. Once these two problem are solved, the continuous absorbing boundary conditions are approximated by discrete absorbing boundary conditions which are then coupled to a standard interior discretization of the differential equations.

Absorbing boundary conditions of such kind have been constructed and analyzed for acoustic and elastic wave propagations by many authors; for detailed expositions, we refer to [5], [6], [8], [9], [11], [20], [21] and the references therein.

The objective of this paper is to develop and analyze both the theoretical perfectly absorbing boundary condition and its local approximations for three-dimensional electromagnetic wave propagation on the boundary of a half-space domain. There are two difficulties in constructing absorbing boundary conditions for the Maxwell system on the boundary of a half-space domain: first, the system is hyperbolic but not strictly hyperbolic; and second, the flat artificial boundary is a uniform characteristic of the system in the sense of [15].

Almost all of the results in this direction that are known to the author were obtained under the assumption that the fields are divergence-free. Under this assumption it is easy to check that each component of both the electric field $\vec{E}$ and the magnetic field $\vec{H}$ satisfies the scalar acoustic wave equation (cf. [3]), for which the absorbing boundary condition theory in the half-space case was already well established by Engquist and Majda [5], [6], Higdon [9], Trefethen and Halpern [21], and others, but it was pointed out by Joly and Mercier [12] that the natural but naive application of this theory to each component of the fields leads to an unstable problem. On the other hand, Duceau and Mercier [4] showed that if one applies the acoustic absorbing boundary conditions only to the tangential components of the electric field, then the resulting problem is stable. Under the same assumption, Joly and Mercier [12] found and analyzed a very interesting and simple second order absorbing boundary condition which only involves first order differential operators and hence can be used easily for the treatment of the edges and corners. When the assumption that the fields are divergence-free is dropped, it seems that the only published result is the one given by Bendali and Halpern [1] in which zero and 
second order absorbing boundary conditions were proposed for the Maxwell system, but it was reported in [17] that their second order absorbing boundary condition leads to an ill-posed problem.

The layout of this paper is as follows. In Section 2, the theoretical perfectly absorbing boundary condition is obtained by considering the Maxwell system as a whole, instead of considering each component of the electromagnetic fields individually. The main idea is to use the Laplace-Fourier transform and construct a special "symmetrizer" or change of dependent variables to derive an implicit formula for the solutions of the Maxwell system. As in the scalar acoustic wave case (cf. [5], [6]), a class of local absorbing boundary conditions could be constructed by using the Padé approximations for the function $\sqrt{1-t^{2}}$, but in this paper we are only interested in its first two approximations. In Sections 3 and 4 reflection and stability properties for the first two local absorbing boundary conditions are analyzed. Finally, the results of some numerical tests are presented in Section 5 to show the performance of these two absorbing boundary conditions.

\section{§2. Derivation of Boundary Conditions}

In this section we first derive the theoretical perfectly absorbing boundary condition in a half-space domain for the Maxwell system. The boundary condition is described by a pseudo-differential equation on the boundary. We then obtain several local and practical absorbing boundary conditions by approximating the pseudo-differential equation near normal incidence.

2.1. Theoretical perfectly absorbing boundary condition. It is well-known that the Maxwell system (1.1) can be simplified by deleting the first two equations if at some time $t_{0}$ (1.1.i) and (1.1.ii) hold simultaneously at each point in space (cf. Dautray and Lions [3] for a proof). Under this condition, the Maxwell system reduces to the following form:

$$
\frac{\partial}{\partial t}\left(\begin{array}{l}
\vec{E} \\
\vec{H}
\end{array}\right)=\left(\begin{array}{cc}
0 & \varepsilon^{-1} \operatorname{curl} \\
-\mu^{-1} \text { curl } & 0
\end{array}\right)\left(\begin{array}{l}
\vec{E} \\
\vec{H}
\end{array}\right)+\left(\begin{array}{l}
\vec{F} \\
\overrightarrow{0}
\end{array}\right) .
$$

If the spatial variables are denoted by $x=\left(x_{1}, x_{2}, x_{3}\right)$, then the curl operator in (2.1) has the representation

$$
\left(\begin{array}{ccc}
0 & 0 & 0 \\
0 & 0 & -1 \\
0 & 1 & 0
\end{array}\right) \frac{\partial}{\partial x_{1}}+\left(\begin{array}{ccc}
0 & 0 & 1 \\
0 & 0 & 0 \\
-1 & 0 & 0
\end{array}\right) \frac{\partial}{\partial x_{2}}+\left(\begin{array}{ccc}
0 & -1 & 0 \\
1 & 0 & 0 \\
0 & 0 & 0
\end{array}\right) \frac{\partial}{\partial x_{3}}
$$

It follows that (2.1) is symmetrically hyperbolic if $\varepsilon=\mu=1$. If $\varepsilon \neq 1$ or $\mu \neq 1$, the exact same symmetric form can be obtained by the following changes of both dependent and independent variables:

$$
x=(\varepsilon \mu)^{-\frac{1}{2}} y, \quad \vec{E}=(\varepsilon \mu)^{-\frac{1}{2}} \vec{D}, \quad \vec{H}=\mu^{-1} \vec{B} .
$$

Hence, in the rest of this paper, unless otherwise stated, we shall always assume that $\varepsilon=\mu=1$. We also assume that $t_{0}=0$ for convenience, and let

$$
\vec{E}(x, 0)=\vec{E}_{0}(x), \quad \vec{H}(x, 0)=\vec{H}_{0}(x),
$$

where $\vec{E}_{0}$ and $\vec{H}_{0}$ are two given vectors which satisfy (1.1.i) and (1.1.ii).

By introducing the six-dimensional vector

$$
\vec{u}(x, t)=\left(u_{1}, u_{2}, u_{3}, u_{4}, u_{5}, u_{6}\right)^{t} \equiv\left(E_{1}, E_{2}, E_{3}, H_{1}, H_{2}, H_{3}\right)^{t}
$$


the Maxwell system (2.1) with (2.4) can be rewritten in the following matrix form:

$$
\begin{gathered}
\frac{\partial \vec{u}}{\partial t}=\sum_{j=1}^{3} A_{j} \frac{\partial \vec{u}}{\partial x_{j}}+\vec{f}, \\
\vec{u}(x, 0)=\vec{u}_{0}(x),
\end{gathered}
$$

where

$$
\begin{gathered}
\vec{u}_{0}(x)=\left(\begin{array}{l}
\vec{E}_{0}(x) \\
\vec{H}_{0}(x)
\end{array}\right) \quad \text { and } \quad A_{j}=\left(\begin{array}{cc}
0 & C_{j} \\
-C_{j} & 0
\end{array}\right), \quad j=1,2,3 ; \\
C_{1}=\left(\begin{array}{ccc}
0 & 0 & 0 \\
0 & 0 & -1 \\
0 & 1 & 0
\end{array}\right), \quad C_{2}=\left(\begin{array}{ccc}
0 & 0 & 1 \\
0 & 0 & 0 \\
-1 & 0 & 0
\end{array}\right), \quad C_{3}=\left(\begin{array}{ccc}
0 & -1 & 0 \\
1 & 0 & 0 \\
0 & 0 & 0
\end{array}\right) .
\end{gathered}
$$

In the definition of $A_{j}$ and later in this paper we abuse the notation by using 0 also to denote the $3 \times 3$ zero matrix. We assume that no confusion will be caused by this.

In this section we consider the Maxwell system (2.5) in the half-space domain $\mathbb{R}_{+}^{3}=\left\{x=\left(x_{1}, x_{2}, x_{3}\right) ; x_{1}>0\right\}$ with the artificial boundary $\Gamma=\{x=$ $\left.\left(x_{1}, x_{2}, x_{3}\right) ; x_{1}=0\right\}$. It is well-known that we need to impose two boundary conditions on $\Gamma$ in order to uniquely determine $\vec{E}$ and $\vec{H}$, and since $\Gamma$ is an artificial boundary, the imposed boundary conditions should not produce too much reflection, and the less reflection the boundary conditions produce, the better boundary conditions they are. The objective of this section is to construct both the theoretical perfectly absorbing boundary condition and its local approximations.

Recall that the Fourier transform of $\vec{u}(x, t)$ with respect to $t$ is defined by

$$
\mathcal{F} \vec{u}(x, \xi)=\int_{-\infty}^{\infty} \vec{u}(x, t) e^{-i \xi t} d t
$$

and the inverse Fourier transform is given by

$$
\vec{u}(x, t)=\frac{1}{2 \pi} \int_{-\infty}^{\infty} \mathcal{F} \vec{u}(x, \xi) e^{i \xi t} d \xi .
$$

The Laplace transform of $\vec{u}(x, t)$ with respect to $t$ is defined by

$$
\mathcal{L} \vec{u}(x, s)=\int_{0}^{\infty} e^{-s t} \vec{u}(x, t) d t=\mathcal{F} \vec{v}(x, t), \quad s=\eta+i \xi(\eta>0),
$$

where

$$
\vec{v}(x, t)= \begin{cases}e^{-\eta t} \vec{u}(x, t), & t>0, \\ 0, & t<0 .\end{cases}
$$

Applying the Fourier transform with respect to both tangential variables $x_{2}$ and $x_{3}$, with dual variables $i \omega_{2}$ and $i \omega_{3}$, and the Laplace transform in $t$, with dual variable $s=\eta+i \xi(\eta>0)$ to system (2.5), the system then becomes

$$
s \overrightarrow{\hat{u}}=A_{1} \frac{\partial \overrightarrow{\hat{u}}}{\partial x_{1}}+i \omega_{2} A_{2} \overrightarrow{\hat{u}}+i \omega_{3} A_{3} \overrightarrow{\hat{u}}+\overrightarrow{\hat{f}}\left(x_{1}, \omega, s\right)+\overrightarrow{\hat{u}}_{0}\left(x_{1}, \omega\right),
$$

where the "hat" denotes the Fourier-Laplace transform and $\omega=\left(\omega_{2}, \omega_{3}\right)$. 
The above equation can also be rewritten as

$$
i A_{1} \frac{\partial \overrightarrow{\hat{u}}}{\partial x_{1}}=M \overrightarrow{\hat{u}}-i \overrightarrow{\hat{g}}
$$

where $\overrightarrow{\hat{g}}=\overrightarrow{\hat{f}}+\overrightarrow{\hat{u}}_{0}$ and $M$ is a $6 \times 6$ matrix which has the following form if we let $I$ denote the $3 \times 3$ identity matrix:

$$
M=M(\omega, s)=\left(\begin{array}{cc}
i s I & M_{1} \\
-M_{1} & i s I
\end{array}\right), \quad M_{1}=\left(\begin{array}{ccc}
0 & -\omega_{3} & \omega_{2} \\
\omega_{3} & 0 & 0 \\
-\omega_{2} & 0 & 0
\end{array}\right) .
$$

Next, we want to decouple the ordinary differential system (2.9) and solve it. To this end we introduce the following change of dependent variable or "symmetrizer" for (2.9), which is a generalization of the one in [15] for the curl operator. In the following we will give a detailed derivation for the case $|\omega| \neq 0$ and a remark for the case $|\omega|=0$ at the end of the derivation.

Let $\omega^{\prime}=\omega /|\omega|,|\omega|^{2}=\omega_{2}^{2}+\omega_{3}^{2}$. Define the linear operator $\mathcal{Q}$ by

$$
\begin{array}{ll}
\mathcal{Q}\left(\vec{e}_{1}\right)=\vec{e}_{4}, & \mathcal{Q}\left(\vec{e}_{4}\right)=\vec{e}_{1}, \\
\mathcal{Q}\left(\vec{e}_{2}\right)=-\omega_{3}^{\prime} \vec{e}_{5}+\omega_{2}^{\prime} \vec{e}_{6}, & \mathcal{Q}\left(\vec{e}_{5}\right)=\omega_{3}^{\prime} \vec{e}_{2}-\omega_{2}^{\prime} \vec{e}_{3}, \\
\mathcal{Q}\left(\vec{e}_{3}\right)=-\omega_{2}^{\prime} \vec{e}_{5}-\omega_{3}^{\prime} \vec{e}_{6}, & \mathcal{Q}\left(\vec{e}_{6}\right)=\omega_{2}^{\prime} \vec{e}_{2}+\omega_{3}^{\prime} \vec{e}_{3},
\end{array}
$$

where $\left\{\vec{e}_{1}, \vec{e}_{2}, \ldots, \vec{e}_{6}\right\}$ is the standard basis of $\mathbb{R}^{6}$. Let $Q$ denote the associate matrix of the operator $\mathcal{Q}$, that is,

$$
\mathcal{Q}\left[\vec{e}_{1}, \vec{e}_{2}, \cdots, \vec{e}_{6}\right]=\left[\vec{e}_{1}, \vec{e}_{2}, \cdots, \vec{e}_{6}\right] Q
$$

then $Q$ is a $6 \times 6$ matrix given by

$$
Q=\left(\begin{array}{cc}
0 & Q_{1} \\
Q_{2} & 0
\end{array}\right), \quad Q_{1}=\left(\begin{array}{ccc}
1 & 0 & 0 \\
0 & \omega_{3}^{\prime} & \omega_{2}^{\prime} \\
0 & -\omega_{2}^{\prime} & \omega_{3}^{\prime}
\end{array}\right), \quad Q_{2}=\left(\begin{array}{ccc}
1 & 0 & 0 \\
0 & -\omega_{3}^{\prime} & -\omega_{2}^{\prime} \\
0 & \omega_{2}^{\prime} & -\omega_{3}^{\prime}
\end{array}\right)
$$

Define the new vector $\overrightarrow{\hat{v}}=Q^{-1} \overrightarrow{\hat{u}}$, where $Q^{-1}$ denotes the inverse matrix of $Q$ and has the form

$$
Q^{-1}=\left(\begin{array}{cc}
0 & Q_{2}^{-1} \\
Q_{1}^{-1} & 0
\end{array}\right), \quad Q_{1}^{-1}=\left(\begin{array}{ccc}
1 & 0 & 0 \\
0 & \omega_{3}^{\prime} & -\omega_{2}^{\prime} \\
0 & \omega_{2}^{\prime} & \omega_{3}^{\prime}
\end{array}\right), \quad Q_{2}^{-1}=\left(\begin{array}{ccc}
1 & 0 & 0 \\
0 & -\omega_{3}^{\prime} & \omega_{2}^{\prime} \\
0 & -\omega_{2}^{\prime} & -\omega_{3}^{\prime}
\end{array}\right)
$$

A direct calculation gives $A_{1} Q^{-1}=Q^{-1} A_{1}$ and

$$
\widetilde{M}=Q^{-1} M Q=\left(\begin{array}{cc}
i s I & \widetilde{M}_{1} \\
\widetilde{M}_{1} & i s I
\end{array}\right), \quad \widetilde{M}_{1}=\left(\begin{array}{ccc}
0 & |\omega| & 0 \\
|\omega| & 0 & 0 \\
0 & 0 & 0
\end{array}\right)
$$

Multiplying equation (2.9) by $i Q^{-1}$, we obtain

$$
-A_{1} \frac{\partial \overrightarrow{\hat{v}}}{\partial x_{1}}=i \widetilde{M} \overrightarrow{\hat{v}}+\overrightarrow{\hat{h}}, \quad \overrightarrow{\hat{h}}=Q^{-1} \overrightarrow{\hat{g}} .
$$


It is easy to check that the corresponding homogeneous system assumes the following form:

$$
\hat{v}_{1}=\frac{i|\omega|}{s} \hat{v}_{5}, \quad \hat{v}_{4}=\frac{i|\omega|}{s} \hat{v}_{2}
$$

$$
\frac{\partial}{\partial x_{1}}\left(\begin{array}{l}
\hat{v}_{2} \\
\hat{v}_{3} \\
\hat{v}_{5} \\
\hat{v}_{6}
\end{array}\right)=\widetilde{N}\left(\begin{array}{c}
\hat{v}_{2} \\
\hat{v}_{3} \\
\hat{v}_{5} \\
\hat{v}_{6}
\end{array}\right)
$$

where

$$
\widetilde{N}=\left(\begin{array}{cccc}
0 & 0 & 0 & -s \\
0 & 0 & s+\frac{|\omega|^{2}}{s} & 0 \\
0 & s & 0 & 0 \\
-\left(s+\frac{|\omega|^{2}}{s}\right) & 0 & 0 & 0
\end{array}\right) .
$$

Since $\operatorname{det}(\lambda I-\widetilde{N})=\left[\lambda^{2}-\left(s^{2}+|\omega|^{2}\right)\right]^{2}, \widetilde{N}$ has two eigenvalues $\lambda_{1}=\sqrt{s^{2}+|\omega|^{2}}$, $\lambda_{2}=-\sqrt{s^{2}+|\omega|^{2}}$, and each is of multiplicity two. The square root branch is chosen so that it is well-defined for $-\pi<\arg z<\pi$ and takes positive values on the real line.

Let

$$
D=\left(\begin{array}{cccc}
\frac{-s}{\lambda_{2}} & 0 & \frac{-s}{\lambda_{1}} & 0 \\
0 & 1 & 0 & 1 \\
0 & \frac{s}{\lambda_{2}} & 0 & \frac{s}{\lambda_{1}} \\
1 & 0 & 1 & 0
\end{array}\right)
$$

then

$$
D^{-1}=\frac{1}{2}\left(\begin{array}{cccc}
\frac{-\lambda_{2}}{s} & 0 & 0 & 1 \\
0 & 1 & \frac{\lambda_{2}}{s} & 0 \\
\frac{-\lambda_{1}}{s} & 0 & 0 & 1 \\
0 & 1 & \frac{\lambda_{1}}{s} & 0
\end{array}\right)
$$

and

$$
D^{-1} \tilde{N} D=\operatorname{diag}\left\{\lambda_{2}, \lambda_{2}, \lambda_{1}, \lambda_{1}\right\} .
$$

Multiplying equation (2.13.ii) by $D^{-1}$, we get

$$
\frac{\partial}{\partial x_{1}}\left(\begin{array}{c}
\hat{w}_{2} \\
\hat{w}_{3} \\
\hat{w}_{5} \\
\hat{w}_{6}
\end{array}\right)=\left(\begin{array}{llll}
\lambda_{2} & & & 0 \\
& \lambda_{2} & & \\
& & \lambda_{1} & \\
0 & & & \lambda_{1}
\end{array}\right)\left(\begin{array}{c}
\hat{w}_{2} \\
\hat{w}_{3} \\
\hat{w}_{5} \\
\hat{w}_{6}
\end{array}\right),
$$

where

$$
\left(\begin{array}{c}
\hat{w}_{2} \\
\hat{w}_{3} \\
\hat{w}_{5} \\
\hat{w}_{6}
\end{array}\right)=D^{-1}\left(\begin{array}{l}
\hat{v}_{2} \\
\hat{v}_{3} \\
\hat{v}_{5} \\
\hat{v}_{6}
\end{array}\right)
$$


The solutions $\left(\hat{w}_{2}, \hat{w}_{3}, \hat{w}_{5}, \hat{w}_{6}\right)$ of (2.14) can be constructed from $e^{\lambda_{1} x_{1}}$ and $e^{\lambda_{2} x_{1}}$, and the solution component $\hat{w}_{k}$, for $k=2,3,5,6$, corresponds to solutions of the Maxwell system (2.5) that are made up of modes

$$
e^{\lambda_{j} x_{1}+i \omega_{2} x_{2}+i \omega_{3} x_{3}+s t}, \quad j=1 \text { or } 2 .
$$

By using a group velocity argument (see Higdon [8] for the details) we know that for $j=1\left(\operatorname{Re} \lambda_{1}>0\right)$ the mode (2.15) is associated with outgoing waves and $e^{\lambda_{1} x_{1}}$ is the outgoing component of the solution, while for $j=2\left(\operatorname{Re} \lambda_{2}<0\right)$ the mode (2.15) is associated with incoming waves and $e^{\lambda_{2} x_{1}}$ is the incoming component of the solution. Thus, the theoretical perfectly absorbing boundary condition is given by

$$
\begin{array}{ll}
\hat{w}_{2}=0, & \text { on } \Gamma, \\
\hat{w}_{3}=0, & \text { on } \Gamma .
\end{array}
$$

Going back to the components of $\hat{u}$, this means that

$$
\begin{aligned}
& \left(\omega_{2}^{\prime} \hat{u}_{2}+\omega_{3}^{\prime} \hat{u}_{3}\right)-\sqrt{1+\frac{|\omega|^{2}}{s^{2}}}\left(\omega_{3}^{\prime} \hat{u}_{5}-\omega_{2}^{\prime} \hat{u}_{6}\right)=0, \quad \text { on } \Gamma, \\
& \left(\omega_{2}^{\prime} \hat{u}_{5}+\omega_{3}^{\prime} \hat{u}_{6}\right)+\sqrt{1+\frac{|\omega|^{2}}{s^{2}}}\left(\omega_{3}^{\prime} \hat{u}_{2}-\omega_{2}^{\prime} \hat{u}_{3}\right)=0, \quad \text { on } \Gamma .
\end{aligned}
$$

Recall that $\hat{u}_{2}=\hat{E}_{2}, \hat{u}_{3}=\hat{E}_{3}, \hat{u}_{5}=\hat{H}_{2}, \hat{u}_{6}=\hat{H}_{3}$, and $s=\eta+i \xi, \eta>0$. Since the above equations hold for any $\eta>0$, letting $\eta \rightarrow 0^{+}$in (2.17) we get the following theoretical perfectly absorbing boundary condition:

$$
\begin{aligned}
& \left(\omega_{2}^{\prime} \hat{E}_{2}+\omega_{3}^{\prime} \hat{E}_{3}\right)-\sqrt{1-\frac{|\omega|^{2}}{\xi^{2}}}\left(\omega_{3}^{\prime} \hat{H}_{2}-\omega_{2}^{\prime} \hat{H}_{3}\right)=0, \quad \text { on } \Gamma, \\
& \left(\omega_{2}^{\prime} \hat{H}_{2}+\omega_{3}^{\prime} \hat{H}_{3}\right)+\sqrt{1-\frac{|\omega|^{2}}{\xi^{2}}}\left(\omega_{3}^{\prime} \hat{E}_{2}-\omega_{2}^{\prime} \hat{E}_{3}\right)=0, \quad \text { on } \Gamma .
\end{aligned}
$$

Remark 2.1. When $|\omega|=0$, the above derivation has to be modified slightly. The first necessary change is that $Q=I$ in $(2.11)$ and $\left(2.13\right.$.i) becomes $\hat{u}_{1}=\hat{u}_{4} \equiv 0$. The second change is that the matrices $\widetilde{N}, D$ and $D^{-1}$ reduce to

$$
\begin{aligned}
\widetilde{N}=\left(\begin{array}{cccc}
0 & 0 & 0 & -s \\
0 & 0 & s & 0 \\
0 & s & 0 & 0 \\
-s & 0 & 0 & 0
\end{array}\right), \quad D=\left(\begin{array}{cccc}
1 & 0 & -1 & 0 \\
0 & 1 & 0 & 1 \\
0 & -1 & 0 & 1 \\
1 & 0 & 1 & 0
\end{array}\right), \\
D^{-1}=\frac{1}{2}\left(\begin{array}{cccc}
1 & 0 & 0 & 1 \\
0 & 1 & -1 & 0 \\
-1 & 0 & 0 & 1 \\
0 & 1 & 1 & 0
\end{array}\right),
\end{aligned}
$$

and the eigenvalues of $\widetilde{N}$ are $\lambda_{1}=s$ and $\lambda_{2}=-s$, and each has multiplicity two. Hence the two conditions in (2.16) become

$$
\begin{array}{ll}
\hat{w}_{2}=\frac{1}{2}\left(\hat{u}_{2}+\hat{u}_{6}\right)=0, & \text { on } \Gamma, \\
\hat{w}_{3}=\frac{1}{2}\left(\hat{u}_{3}-\hat{u}_{5}\right)=0, & \text { on } \Gamma .
\end{array}
$$


That is,

$$
\begin{array}{ll}
\hat{E}_{2}\left(x_{1}, 0, s\right)+\hat{H}_{3}\left(x_{1}, 0, s\right)=0, & \text { on } \Gamma, \\
\hat{E}_{3}\left(x_{1}, 0, s\right)-\hat{H}_{2}\left(x_{1}, 0, s\right)=0, & \text { on } \Gamma .
\end{array}
$$

Remark 2.2. We remark that the (2.18.iii) and (2.18.iv) only hold when $\omega=\left(\omega_{1}, \omega_{2}\right)$ $=0$ in the above derivation. On the other hand, a slight stretch of these conditions leads to the following observation. After making a trivial extension (i.e., we let the extended functions satisfy the same equation for all $\omega$ ) we get the following zeroth order differential equations in the time-domain:

$$
\begin{array}{ll}
E_{2}+H_{3}=0, & \text { on } \Gamma, \\
E_{3}-H_{2}=0, & \text { on } \Gamma,
\end{array}
$$

which we refer as our zeroth order absorbing boundary condition.

2.2. Local absorbing boundary conditions. Obviously, the theoretical perfectly absorbing boundary condition (2.18) is nonlocal in both space and time. This boundary condition is impractical from a computational point of view, since to advance one time level at a single point requires information from all previous times over the entire boundary. Hence it is necessary and useful to have local approximations to the perfectly absorbing boundary condition. To derive practical absorbing boundary conditions, we will adopt the following three criteria of Engquist and Majda (cf. [5], [6]):

(1) The boundary conditions substantially reduce the (unphysical) reflections from the artificial boundary.

(2) The boundary conditions are local.

(3) Each of the boundary conditions together with the interior differential equation defines a well-posed mixed boundary value problem.

In the rest of this section, we only construct several local absorbing boundary conditions, and leave the verification of the above criteria (1) and (3) for the next two sections.

Since for a real vector $\vec{u}(x, t)$, the Fourier transform $\mathcal{F} \vec{u}(x, \xi)$ with respect to $t$ satisfies

$$
\mathcal{F} \vec{u}(x,-\xi)=\overline{\mathcal{F} \vec{u}(x, \xi)}, \quad \xi \in \mathbb{R},
$$

the inverse Fourier transform takes the form

$$
\vec{u}(x, t)=\frac{1}{\pi} \operatorname{Re} \int_{0}^{\infty} \mathcal{F} \vec{u}(x, \xi) e^{i \xi t} d \xi .
$$

Hence, in the following we assume $\xi>0$.

Similarly to the construction for the scalar acoustic wave equation [5], our local absorbing boundary conditions result from the Padé approximation of $\sqrt{1-|\omega|^{2} / \xi^{2}}$. Other types of approximation also could be used (see Trefethen and Halpern [21]), but we shall not discuss them here.

We approximate $\sqrt{1-|\omega|^{2} / \xi^{2}}$ at normal incidence $|\omega|=0$. The first approximation is $\sqrt{1-|\omega|^{2} / \xi^{2}}=1+O\left(|\omega|^{2} / \xi^{2}\right)$. So after replacing $\sqrt{1-|\omega|^{2} / \xi^{2}}$ by 1 in (2.18.i) and (2.18.ii) and applying the inverse Fourier transform to the resulting equations, we obtain the following first order local absorbing boundary conditions 
for the Maxwell system (2.5) with $\vec{f}=0$ :

$$
\begin{aligned}
& \frac{\partial E_{2}}{\partial x_{2}}+\frac{\partial E_{3}}{\partial x_{3}}-\left(\frac{\partial H_{2}}{\partial x_{3}}-\frac{\partial H_{3}}{\partial x_{2}}\right)=0, \quad \text { on } \Gamma, \\
& \left(\frac{\partial E_{2}}{\partial x_{3}}-\frac{\partial E_{3}}{\partial x_{2}}\right)+\frac{\partial H_{2}}{\partial x_{2}}+\frac{\partial H_{3}}{\partial x_{3}}=0, \quad \text { on } \Gamma .
\end{aligned}
$$

Since the Maxwell system originally holds on the artificial boundary, we can use this fact to simplify the above first order absorbing boundary conditions. By the first and fourth equations in the Maxwell system (2.1), the above two boundary conditions can be simplified as follows:

$$
\begin{array}{ll}
\frac{\partial E_{1}}{\partial t}+\frac{\partial E_{2}}{\partial x_{2}}+\frac{\partial E_{3}}{\partial x_{3}}=0, & \text { on } \Gamma, \\
\frac{\partial H_{1}}{\partial t}+\frac{\partial H_{2}}{\partial x_{2}}+\frac{\partial H_{3}}{\partial x_{3}}=0, & \text { on } \Gamma .
\end{array}
$$

Similarly, the second approximation (the first Taylor or Padé) to the square root, $\sqrt{1-|\omega|^{2} / \xi^{2}}=1-|\omega|^{2} / 2 \xi^{2}+O\left(|\omega|^{4} / \xi^{4}\right)$, leads to the following second order local absorbing boundary condition:

$$
\begin{array}{r}
\frac{\partial^{2}}{\partial t^{2}}\left[\frac{\partial E_{2}}{\partial x_{2}}+\frac{\partial E_{3}}{\partial x_{3}}-\left(\frac{\partial H_{2}}{\partial x_{3}}-\frac{\partial H_{3}}{\partial x_{2}}\right)\right]+\frac{1}{2}\left(\frac{\partial^{2}}{\partial x_{2}^{2}}+\frac{\partial^{2}}{\partial x_{3}^{2}}\right)\left(\frac{\partial H_{2}}{\partial x_{3}}-\frac{\partial H_{3}}{\partial x_{2}}\right)=0, \\
-\frac{1}{2}\left(\frac{\partial^{2}}{\partial x_{2}^{2}}+\frac{\partial^{2}}{\partial x_{3}^{2}}\right)\left(\frac{\partial E_{2}}{\partial x_{3}}-\frac{\partial E_{3}}{\partial x_{2}}\right)+\frac{\partial^{2}}{\partial t^{2}}\left[\left(\frac{\partial E_{2}}{\partial x_{3}}-\frac{\partial E_{3}}{\partial x_{2}}\right)+\frac{\partial H_{2}}{\partial x_{2}}+\frac{\partial H_{3}}{\partial x_{3}}\right]=0, \\
\text { on } \Gamma .
\end{array}
$$

Again, by the first and fourth equations in the Maxwell system (2.1), the above two boundary conditions can be simplified as follows:

$$
\begin{array}{ll}
\frac{\partial}{\partial t}\left(\frac{\partial E_{1}}{\partial t}+\frac{\partial E_{2}}{\partial x_{2}}+\frac{\partial E_{3}}{\partial x_{3}}\right)-\frac{1}{2}\left(\frac{\partial^{2} E_{1}}{\partial x_{2}^{2}}+\frac{\partial^{2} E_{1}}{\partial x_{3}^{2}}\right)=0, & \text { on } \Gamma, \\
\frac{\partial}{\partial t}\left(\frac{\partial H_{1}}{\partial t}+\frac{\partial H_{2}}{\partial x_{2}}+\frac{\partial H_{3}}{\partial x_{3}}\right)-\frac{1}{2}\left(\frac{\partial^{2} H_{1}}{\partial x_{2}^{2}}+\frac{\partial^{2} H_{1}}{\partial x_{3}^{2}}\right)=0, & \text { on } \Gamma .
\end{array}
$$

We could continue to construct higher order local absorbing boundary conditions for the Maxwell system, but since higher order absorbing boundary conditions are used less frequently due to difficulties of numerical implementation, they will not be given here. We conclude this section by noticing that when the electric and magnetic fields are divergence-free, i.e., $\operatorname{div} \vec{E}=\operatorname{div} \vec{H}=0$, the above first and second order absorbing boundary conditions can be rewritten as

$$
\begin{aligned}
& \frac{\partial E_{1}}{\partial t}-\frac{\partial E_{1}}{\partial x_{1}}=0, \quad \text { on } \Gamma, \\
& \frac{\partial H_{1}}{\partial t}-\frac{\partial H_{1}}{\partial x_{1}}=0, \quad \text { on } \Gamma,
\end{aligned}
$$

and

$$
\begin{aligned}
& \frac{\partial^{2} E_{1}}{\partial t^{2}}-\frac{\partial^{2} E_{1}}{\partial t \partial x_{1}}-\frac{1}{2}\left(\frac{\partial^{2} E_{1}}{\partial x_{2}^{2}}+\frac{\partial^{2} E_{1}}{\partial x_{3}^{2}}\right)=0, \quad \text { on } \Gamma, \\
& \frac{\partial^{2} H_{1}}{\partial t^{2}}-\frac{\partial^{2} H_{1}}{\partial t \partial x_{1}}-\frac{1}{2}\left(\frac{\partial^{2} H_{1}}{\partial x_{2}^{2}}+\frac{\partial^{2} H_{1}}{\partial x_{3}^{2}}\right)=0, \quad \text { on } \Gamma \text {. }
\end{aligned}
$$


This is the same as applying the classical first and second order Engquist-Majda (see [5], [6]) absorbing boundary operators for the scalar acoustic wave equation to the normal components $E_{1}$ and $H_{1}$ of the electromagnetic fields on $\Gamma$.

Remark 2.3. It is easy to check that if $\epsilon \neq 1$ and $\mu \neq 1$, the boundary conditions (2.20) and (2.21) should be replaced by the following pair of boundary conditions:

$$
\begin{array}{ll}
\frac{1}{c} \frac{\partial E_{1}}{\partial t}+\frac{\partial E_{2}}{\partial x_{2}}+\frac{\partial E_{3}}{\partial x_{3}}=0, & \text { on } \Gamma, \\
\frac{1}{c} \frac{\partial H_{1}}{\partial t}+\frac{\partial H_{2}}{\partial x_{2}}+\frac{\partial H_{3}}{\partial x_{3}}=0, & \text { on } \Gamma,
\end{array}
$$

and

$$
\begin{array}{ll}
\frac{\partial}{\partial t}\left(\frac{1}{c} \frac{\partial E_{1}}{\partial t}+\frac{\partial E_{2}}{\partial x_{2}}+\frac{\partial E_{3}}{\partial x_{3}}\right)-\frac{c}{2}\left(\frac{\partial^{2} E_{1}}{\partial x_{2}^{2}}+\frac{\partial^{2} E_{1}}{\partial x_{3}^{2}}\right)=0, & \text { on } \Gamma \\
\frac{\partial}{\partial t}\left(\frac{1}{c} \frac{\partial H_{1}}{\partial t}+\frac{\partial H_{2}}{\partial x_{2}}+\frac{\partial H_{3}}{\partial x_{3}}\right)-\frac{c}{2}\left(\frac{\partial^{2} H_{1}}{\partial x_{2}^{2}}+\frac{\partial^{2} H_{1}}{\partial x_{3}^{2}}\right)=0, & \text { on } \Gamma,
\end{array}
$$

where $c=1 / \sqrt{\epsilon \mu}$ is the speed of the electromagnetic wave.

In the next section we will show that the boundary conditions (2.20) and (2.21) annihilate plane waves traveling out of the domain $\mathbb{R}_{+}^{3}$ normally to the boundary, but they do produce some reflections for plane waves traveling out of the domain at nonzero angles of incidence. To completely absorb plane waves of nonzero angle of incidence, we use an idea of Higdon (cf. [9], [11]) to propose the following variations of the boundary conditions $(2.20)$ and (2.21):

$$
\begin{array}{ll}
(\cos \beta) \frac{\partial E_{1}}{\partial t}+\frac{\partial E_{2}}{\partial x_{2}}+\frac{\partial E_{3}}{\partial x_{3}}=0, & \text { on } \Gamma, \\
(\cos \beta) \frac{\partial H_{1}}{\partial t}+\frac{\partial H_{2}}{\partial x_{2}}+\frac{\partial H_{3}}{\partial x_{3}}=0, & \text { on } \Gamma,
\end{array}
$$

and

$$
\begin{aligned}
& {\left[1+\left(\cos \beta_{1}\right)\left(\cos \beta_{2}\right)\right] \frac{\partial^{2} E_{1}}{\partial t^{2}}+} {\left[\left(\cos \beta_{1}\right)+\left(\cos \beta_{2}\right)\right]\left(\frac{\partial^{2} E_{2}}{\partial t \partial x_{2}}+\frac{\partial^{2} E_{3}}{\partial t \partial x_{3}}\right) } \\
&-\left(\frac{\partial^{2} E_{1}}{\partial x_{2}^{2}}+\frac{\partial^{2} E_{1}}{\partial x_{3}^{2}}\right)=0, \quad \text { on } \Gamma, \\
& {\left[1+\left(\cos \beta_{1}\right)\left(\cos \beta_{2}\right)\right] \frac{\partial^{2} H_{1}}{\partial t^{2}}+} {\left[\left(\cos \beta_{1}\right)+\left(\cos \beta_{2}\right)\right]\left(\frac{\partial^{2} H_{2}}{\partial t \partial x_{2}}+\frac{\partial^{2} H_{3}}{\partial t \partial x_{3}}\right) } \\
&-\left(\frac{\partial^{2} H_{1}}{\partial x_{2}^{2}}+\frac{\partial^{2} H_{1}}{\partial x_{3}^{2}}\right)=0, \quad \text { on } \Gamma .
\end{aligned}
$$

We will show in the next section that the new boundary condition (2.26) annihilates plane waves traveling out of the domain $\mathbb{R}_{+}^{3}$ with angles of incidence $\pm \beta$, while the condition (2.27) annihilates outgoing plane waves with angles of incidence $\pm \beta_{1}$ and $\pm \beta_{2}$.

\section{§3. Properties of the local absorbing boundary Conditions}

In this section we give two properties of the first and second order local absorbing boundary conditions which we developed in the last section. First, we examine the reflection property of each of these two local boundary conditions at $\Gamma$. Then, we 
briefly indicate the invariance of these boundary conditions with respect to any rotation of the tangential coordinates $\left(x_{2}, x_{3}\right)$.

To analyze the reflection properties of the boundary conditions (2.20) and (2.21), we are interested in determining the sizes of the reflection coefficients when sinusoidal plane waves of the following homogeneous Maxwell system strike the boundary $\Gamma$ :

$$
\begin{aligned}
\frac{\partial \vec{E}}{\partial t} & =\operatorname{curl} \vec{H}, \\
\frac{\partial \vec{H}}{\partial t} & =-\operatorname{curl} \vec{E}, \\
\operatorname{div} \vec{E} & =0, \quad \operatorname{div} \vec{H}=0 .
\end{aligned}
$$

It is well-known (see [14], [19]) that (3.1) admits plane wave solutions of the form

$$
\left(\begin{array}{l}
\vec{E} \\
\vec{H}
\end{array}\right)=\left(\begin{array}{c}
\vec{E}^{0} \\
\vec{H}^{0}
\end{array}\right) e^{i(\alpha \cdot x+\xi t)}
$$

for any $\alpha=\left(\alpha_{1}, \alpha_{2}, \alpha_{3}\right)^{t} \in \mathbb{R}^{3}$ and $\xi \in \mathbb{R}$ that satisfy the dispersion relation

$$
\xi^{2}=|\alpha|^{2}, \quad|\alpha|=\sqrt{\alpha_{1}^{2}+\alpha_{2}^{2}+\alpha_{3}^{2}} .
$$

Let $\alpha^{\prime}=\alpha / \xi$; then $\left|\alpha^{\prime}\right|=1$, and (3.2) becomes

$$
\left(\begin{array}{l}
\vec{E} \\
\vec{H}
\end{array}\right)=\left(\begin{array}{c}
\vec{E}^{0} \\
\vec{H}^{0}
\end{array}\right) e^{i \xi\left(\alpha^{\prime} \cdot x+t\right)}
$$

Substituting (3.4) into (3.1.i)-(3.1.iii) we have that $\vec{E}^{0}$ and $\vec{H}^{0}$ must satisfy the following two equations (cf. [19] for a proof):

$$
\begin{aligned}
\alpha^{\prime} \cdot \vec{E}^{0} & =0, & \alpha^{\prime} \cdot \vec{H}^{0} & =0 . \\
\alpha^{\prime} \times \vec{E}^{0} & =-\vec{H}^{0}, & \alpha^{\prime} \times \vec{H}^{0} & =\vec{E}^{0} .
\end{aligned}
$$

From (3.5) we see that both $\vec{E}$ and $\vec{H}$ are perpendicular to the wave propagation vector $\alpha^{\prime}$, so this wave is a transverse electromagnetic wave (a TEM wave).

Let $\theta$ denote the angle between the propagation direction $\alpha^{\prime}$ and the positive $x_{1}$-axis; then $\alpha_{1}^{\prime}=\cos \theta$. Since we are interested in the reflection of the above incident plane waves at the artificial boundary $\Gamma$, we only need to consider the case $|\theta|<\frac{\pi}{2}, \alpha_{1}^{\prime}=\cos \theta>0$.

For $\alpha^{\prime}=\left(\alpha_{1}^{\prime}, \alpha_{2}^{\prime}, \alpha_{3}^{\prime}\right)^{t}, \alpha_{1}^{\prime}>0,\left|\alpha^{\prime}\right|=1$, let $\vec{E}^{0}=\left(E_{1}^{0}, E_{2}^{0}, E_{3}^{0}\right)^{t}$ be a vector satisfying the first equation in (3.5), i.e., $\alpha^{\prime} \cdot \vec{E}^{0}=0$. By the first equation in (3.6) we get

$$
\vec{H}^{0}=-\alpha^{\prime} \times \vec{E}^{0}=\left(\begin{array}{c}
E_{2}^{0} \alpha_{3}^{\prime}-E_{3}^{0} \alpha_{2}^{\prime} \\
E_{3}^{0} \alpha_{1}^{\prime}-E_{1}^{0} \alpha_{3}^{\prime} \\
E_{1}^{0} \alpha_{2}^{\prime}-E_{2}^{0} \alpha_{1}^{\prime}
\end{array}\right) .
$$

In order to measure the reflection produced by the local boundary conditions (2.20) and (2.21) when a sinusoidal plane wave strikes the boundary $\Gamma$, we consider 
solutions of the interior equation (3.1) which satisfy (2.20) or (2.21) and have the forms:

$$
\begin{aligned}
& \vec{E}=\vec{E}_{I}+\vec{E}_{R}, \\
& \vec{H}=\vec{H}_{I}+\vec{H}_{R},
\end{aligned}
$$

where the incident waves $\vec{E}_{I}, \vec{H}_{I}$ and the reflected waves $\vec{E}_{R}, \vec{H}_{R}$ have the following forms:

$$
\begin{gathered}
\vec{E}_{I}=\vec{E}^{0} e^{i \xi\left(\alpha_{1}^{\prime} x_{1}+\alpha_{2}^{\prime} x_{2}+\alpha_{3}^{\prime} x_{3}+t\right)}, \quad \vec{H}_{I}=\vec{H}^{0} e^{i \xi\left(\alpha_{1}^{\prime} x_{1}+\alpha_{2}^{\prime} x_{2}+\alpha_{3}^{\prime} x_{3}+t\right)}, \\
\vec{E}_{R}=R_{c}\left(\begin{array}{c}
-E_{1}^{0} \\
E_{2}^{0} \\
E_{3}^{0}
\end{array}\right) e^{i \xi\left(-\alpha_{1}^{\prime} x_{1}+\alpha_{2}^{\prime} x_{2}+\alpha_{3}^{\prime} x_{3}+t\right)}, \\
\vec{H}_{R}=R_{c}\left(\begin{array}{c}
-H_{1}^{0} \\
H_{2}^{0} \\
H_{3}^{0}
\end{array}\right) e^{i \xi\left(-\alpha_{1}^{\prime} x_{1}+\alpha_{2}^{\prime} x_{2}+\alpha_{3}^{\prime} x_{3}+t\right)},
\end{gathered}
$$

where $R_{c}$ is the reflection coefficient to be computed.

The objective of the present analysis is to determine the magnitude of the reflection coefficient $R_{c}$ when the boundary conditions defined by (2.20) or (2.21) are imposed. The desired boundary conditions should produce smaller $R_{c}$, so we want $R_{c}$ to be as small as possible. Introducing $\ell=\alpha_{2}^{\prime} x_{2}+\alpha_{3}^{\prime} x_{3}+t$ and substituting (3.8) (with (3.9)) into (2.20), we obtain

$$
\begin{array}{ll}
i \xi\left[\left(E_{1}^{0}+E_{2}^{0} \alpha_{2}^{\prime}+E_{3}^{0} \alpha_{3}^{\prime}\right)+R_{c}\left(-E_{1}^{0}+E_{2}^{0} \alpha_{2}^{\prime}+E_{3}^{0} \alpha_{3}^{\prime}\right)\right] e^{i \xi \ell}=0, & \text { on } \Gamma . \\
i \xi\left[\left(H_{1}^{0}+H_{2}^{0} \alpha_{2}^{\prime}+H_{3}^{0} \alpha_{3}^{\prime}\right)+R_{c}\left(-H_{1}^{0}+H_{2}^{0} \alpha_{2}^{\prime}+H_{3}^{0} \alpha_{3}^{\prime}\right)\right] e^{i \xi \ell}=0, & \text { on } \Gamma .
\end{array}
$$

Noticing that $\vec{E}^{0} \cdot \alpha^{\prime}=E_{1}^{0} \alpha_{1}^{\prime}+E_{2}^{0} \alpha_{2}^{\prime}+E_{3}^{0} \alpha_{3}^{\prime}=0$ and $\vec{H}^{0}$ satisfies (3.7), which implies that $\vec{H}^{0} \cdot \alpha^{\prime}=0$, we obtain from (3.10) and (3.11) that

$$
R_{c}^{(1)}=\frac{1-\alpha_{1}^{\prime}}{1+\alpha_{1}^{\prime}}=\frac{1-\cos \theta}{1+\cos \theta},
$$

where the superscript (1) indicates that $R_{c}^{(1)}$ is the reflection coefficient corresponding to the first order absorbing boundary condition (2.20).

To determine $R_{c}^{(2)}$, we substitute (3.8) (with (3.9)) into (2.21) and get

$(i \xi)^{2}\left\{\left[\left(E_{1}^{0}+E_{2}^{0} \alpha_{2}^{\prime}+E_{3}^{0} \alpha_{3}^{\prime}\right)+R_{c}\left(-E_{1}^{0}+E_{2}^{0} \alpha_{2}^{\prime}+E_{3}^{0} \alpha_{3}^{\prime}\right)\right]\right.$.

$$
\left.-\frac{1}{2}\left(1-R_{c}\right) E_{1}^{0}\left[\left(\alpha_{2}^{\prime}\right)^{2}+\left(\alpha_{3}^{\prime}\right)^{2}\right]\right\} e^{i \xi \ell}=0, \quad \text { on } \Gamma .
$$

$(i \xi)^{2}\left\{\left[\left(H_{1}^{0}+H_{2}^{0} \alpha_{2}^{\prime}+H_{3}^{0} \alpha_{3}^{\prime}\right)+R_{c}\left(-H_{1}^{0}+H_{2}^{0} \alpha_{2}^{\prime}+H_{3}^{0} \alpha_{3}^{\prime}\right)\right]\right.$

$$
\left.-\frac{1}{2}\left(1-R_{c}\right) H_{1}^{0}\left[\left(\alpha_{2}^{\prime}\right)^{2}+\left(\alpha_{3}^{\prime}\right)^{2}\right]\right\} e^{i \xi \ell}=0, \quad \text { on } \Gamma .
$$

Recalling that $\alpha^{\prime} \cdot E^{0}=\alpha^{\prime} \cdot H^{0}=0$ and $\left(\alpha_{1}^{\prime}\right)^{2}=1-\left(\alpha_{2}^{\prime}\right)^{2}-\left(\alpha_{3}^{\prime}\right)^{2}$, we get from (2.13) and (2.14) that

$$
R_{c}^{(2)}=\left(\frac{1-\alpha_{1}^{\prime}}{1+\alpha_{1}^{\prime}}\right)^{2}=\left(\frac{1-\cos \theta}{1+\cos \theta}\right)^{2} .
$$


Summarizing the above derivation, we have the first main theorem of this paper.

Theorem 3.1. Any electromagnetic plane wave of the form (3.4) which travels out of the domain $\mathbb{R}_{+}^{3}$ at the angle of incidence $\theta,|\theta|<\frac{\pi}{2}$, is reflected at $\Gamma$ by the boundary conditions (2.20) and (2.21) respectively into another plane wave which has the same polarization as the "outgoing wave" does and whose amplitude equals the amplitude of the "outgoing wave" times the reflection coefficient

$$
R_{c}^{(1)}(\theta)=\frac{1-\cos \theta}{1+\cos \theta} \quad \text { or } \quad R_{c}^{(2)}(\theta)=\left(\frac{1-\cos \theta}{1+\cos \theta}\right)^{2},
$$

respectively.

Remark 3.1. Following the same derivation one can show that the reflection coefficients of the boundary conditions (2.26) and (2.27) are given by

$$
\tilde{R}_{c}^{(1)}(\theta)=\frac{\cos \beta-\cos \theta}{\cos \beta+\cos \theta} \quad \text { and } \quad \tilde{R}_{c}^{(2)}(\theta)=\frac{\cos \beta_{1}-\cos \theta}{\cos \beta_{1}+\cos \theta} \cdot \frac{\cos \beta_{2}-\cos \theta}{\cos \beta_{2}+\cos \theta},
$$

respectively. So the boundary condition (2.26) annihilates plane waves traveling out of the domain $\mathbb{R}_{+}^{3}$ with angles of incidence $\pm \beta$, while the condition (2.27) annihilates outgoing plane waves with angles of incidence $\pm \beta_{1}$ and $\pm \beta_{2}$.

Remark 3.2. In [12], Joly and Mercier showed that their second order absorbing boundary condition for the Maxwell system is invariant with respect to any rotation about the normal axis. This is another very important property for an absorbing boundary condition to have, since the same property is already possessed by the Maxwell system in the interior domain. Here we remark that our local absorbing boundary conditions $(2.20),(2.21),(2.26)$ and $(2.27)$ also possess this invariance property.

To see this, we notice that the only places the tangential coordinates $x_{2}$ and $x_{3}$ appear in the boundary conditions $(2.20),(2.21),(2.26)$ and $(2.27)$ are either in the divergences $\frac{\partial E_{2}}{\partial x_{2}}+\frac{\partial E_{3}}{\partial x_{3}}$ and $\frac{\partial H_{2}}{\partial x_{2}}+\frac{\partial H_{3}}{\partial x_{3}}$, or in the Laplacians $\frac{\partial^{2} E_{1}}{\partial x_{2}^{2}}+\frac{\partial^{2} E_{1}}{\partial x_{3}^{2}}$ and

$\frac{\partial^{2} H_{1}}{\partial x_{2}^{2}}+\frac{\partial^{2} H_{1}}{\partial x_{3}^{2}}$. Hence, any rotation about the normal axis, which is a rotation in the $\left(x_{2}, x_{3}\right)$ plane, will not change the boundary conditions $(2.20),(2.21),(2.26)$ and (2.27), since both the divergence operator and the Laplace operator are invariant under rotations (cf. [14]).

\section{§4. AnALYsis OF STABILITY OF LOCAL ABSORBING BOUNDARY CONDITIONS}

In this section, we will show that the boundary conditions introduced in the previous section satisfy a stability condition originally developed by Kreiss and Majda-Osher for determining well-posedness of initial-boundary value problems for first order linear noncharacteristic strictly hyperbolic systems (cf. [13]) and uniformly characteristic hyperbolic systems (cf. [15]). This condition is often called the Kreiss criterion or Kreiss condition, and when it is satisfied we say that the initial-boundary value problem is well-posed in the sense of Kreiss. Recall that the Maxwell system is not strictly hyperbolic and the flat boundary $\Gamma=\{x=$ $\left.\left(x_{1}, x_{2}, x_{3}\right) ; x_{1}=0\right\}$ of the half-space $\mathbb{R}_{+}^{3}=\left\{x=\left(x_{1}, x_{2}, x_{3}\right) ; x_{1}>0\right\}$ domain is a uniform characteristic of the system in the sense of [15]. 
4.1. Verification of the Kreiss condition. We recall the following:

Kreiss condition. For an initial-boundary value problem defined on the half space domain $\mathbb{R}_{+}^{3}$, a boundary condition on $\Gamma$ is said to fulfill the Kreiss condition if and only if the boundary condition is not satisfied by any nonzero solution of the interior equation belonging to either of the following categories:

(i) Solutions of the form

$$
\vec{\varphi}\left(x_{1}\right) e^{i \omega_{2} x_{2}+i \omega_{3} x_{3}+s t}
$$

for which $\operatorname{Re} s>0$ and $\vec{\varphi}\left(x_{1}\right) \rightarrow 0$ as $x_{1} \rightarrow+\infty$.

(ii) Solutions that are limits of solutions in (i) as $\operatorname{Re} s \rightarrow 0^{+}$.

Remark 4.1. The Kreiss condition in the above form is due to Higdon for the noncharacteristic case (page 851 of [11]), and the same description was given by Majda and Osher for the uniformly characteristic case (page 614 of [15]). This is an equivalent formulation of the original Kreiss condition; we refer to [8],[11] for a discussion on this and also for a physical interpretation of above the Kreiss condition. For the original algebraic description of the Kreiss condition, we refer to Kreiss [13] and to Majda and Osher [15].

The objective of this subsection is to verify that the local absorbing boundary conditions introduced in the last section satisfy the Kreiss condition. Before we do this, we first need to prove the following characterization theorem for decaying solutions of the form (4.1) of the homogeneous Maxwell system.

Theorem 4.1. If $\operatorname{Re} s \geq 0$, then a nonzero solution $\vec{u}$ of the homogeneous Maxwell system

$$
\frac{\partial \vec{u}}{\partial t}=\sum_{j=1}^{3} A_{j} \frac{\partial \vec{u}}{\partial x_{j}}, \quad \vec{u}=\left(\begin{array}{l}
\vec{E} \\
\vec{H}
\end{array}\right),
$$

of the form (4.1) tends to zero as $x_{1} \rightarrow+\infty$ if and only if $\vec{u}$ assumes the following form:

$$
\vec{u}=\vec{\varphi}\left(x_{1}\right) e^{i \omega_{2} x_{2}+i \omega_{3} x_{3}+s t},
$$

where

$$
\begin{aligned}
\vec{\varphi}\left(x_{1}\right) & =\left(c_{3} \vec{a}_{2}+c_{4} \vec{b}_{2}\right) e^{\lambda_{2} x_{1}}, \quad \lambda_{2}=-\sqrt{s^{2}+|\omega|^{2}}, \\
|\omega|^{2} & =\omega_{2}^{2}+\omega_{3}^{2}, \quad \omega=\left(\omega_{2}, \omega_{3}\right), \quad \omega^{\prime}=\frac{\omega}{|\omega|}, \\
\vec{a}_{2} & = \begin{cases}\left(\frac{-i|\omega|}{\lambda_{2}}, \omega_{2}^{\prime}, \omega_{3}^{\prime}, 0, \frac{\omega_{3}^{\prime} s}{\lambda_{2}},-\frac{\omega_{2}^{\prime} s}{\lambda_{2}}\right)^{t}, & \text { if }|\omega| \neq 0, \\
(0,1,0,0,0,1)^{t}, & \text { if }|\omega|=0, s \neq 0,\end{cases} \\
\vec{b}_{2} & = \begin{cases}\left(0, \frac{\omega_{3}^{\prime} s}{\lambda_{2}},-\frac{\omega_{2}^{\prime} s}{\lambda_{2}}, \frac{i|\omega|}{\lambda_{2}},-\omega_{2}^{\prime},-\omega_{3}^{\prime}\right)^{t}, & \text { if }|\omega| \neq 0, \\
(0,0,1,0,-1,0)^{t}, & \text { if }|\omega|=0, s \neq 0,\end{cases}
\end{aligned}
$$

where $c_{3}$ and $c_{4}$ are two arbitrary complex constants such that $\left|c_{3}\right|+\left|c_{4}\right| \neq 0$.

Proof. Let $\vec{u}=\vec{\varphi}\left(x_{1}\right) e^{i \omega_{2} x_{2}+i \omega_{3} x_{3}+s t}$ be a solution of (4.2). Substituting $\vec{u}$ into (4.2), we get

$$
i A_{1} \frac{\partial \vec{\varphi}\left(x_{1}\right)}{\partial x_{1}}=M(\omega, s) \vec{\varphi}\left(x_{1}\right),
$$

where $M(\omega, s)$ is defined by $(2.10)$. 
First, we consider the case $|\omega|=0$. Notice that $M(0, s)=i s I$, so the ordinary differential system (4.4) reduces to the following three decoupled $2 \times 2$ systems:

$$
\left\{\begin{array} { l } 
{ 0 = s \vec { \varphi } _ { 1 } ( x _ { 1 } ) , } \\
{ 0 = s \vec { \varphi } _ { 4 } ( x _ { 1 } ) , }
\end{array} \quad \left\{\begin{array} { l } 
{ \vec { \varphi } _ { 2 } ( x _ { 1 } ) = - s \vec { \varphi } _ { 6 } ( x _ { 1 } ) , } \\
{ \vec { \varphi } _ { 6 } ( x _ { 1 } ) = - s \vec { \varphi } _ { 2 } ( x _ { 1 } ) , }
\end{array} \quad \left\{\begin{array}{l}
\vec{\varphi}_{3}\left(x_{1}\right)=-s \vec{\varphi}_{5}\left(x_{1}\right), \\
\vec{\varphi}_{5}\left(x_{1}\right)=-s \vec{\varphi}_{3}\left(x_{1}\right) .
\end{array}\right.\right.\right.
$$

Clearly, when $s=0$ (4.4) only has constant (in $x_{1}$ ) solutions. Consequently, (4.2) has no decaying solutions of the form (4.1) in this case. On the other hand, when $s \neq 0$, the general solution of (4.4) has the form

$$
\vec{\varphi}\left(x_{1}\right)=\left(c_{1} \vec{a}_{1}+c_{2} \vec{b}_{1}\right) e^{\lambda_{1} x_{1}}+\left(c_{3} \vec{a}_{2}+c_{4} \vec{b}_{2}\right) e^{\lambda_{2} x_{1}},
$$

where

$$
\begin{array}{ll}
\vec{a}_{1}=(0,1,0,0,0,-1)^{t}, & \vec{b}_{1}=(0,0,1,0,1,0)^{t}, \\
\vec{a}_{2}=(0,1,0,0,0,1)^{t}, & \vec{b}_{2}=(0,0,1,0,-1,0)^{t},
\end{array}
$$

and $c_{1}, \cdots, c_{4}$ are arbitrary complex constants. The general solution of the form (4.1) of the equation (4.2), therefore, is

$$
\vec{u}=\left[\left(c_{1} \vec{a}_{1}+c_{2} \vec{b}_{1}\right) e^{\lambda_{1} x_{1}}+\left(c_{3} \vec{a}_{2}+c_{4} \vec{b}_{2}\right) e^{\lambda_{2} x_{1}}\right] e^{i \omega_{2} x_{2}+i \omega_{3} x_{3}+s t} .
$$

Since $\operatorname{Re} \lambda_{1}>0$ and $\operatorname{Re} \lambda_{2}<0,\left|e^{\lambda_{1} x_{1}}\right| \rightarrow+\infty$ and $\left|e^{\lambda_{2} x_{1}}\right| \rightarrow 0$ as $x_{1} \rightarrow+\infty$. Noticing that $\vec{a}_{1}$ and $\vec{b}_{1}$ are linearly independent, we conclude that (nonzero) $\vec{u} \rightarrow 0$ as $x_{1} \rightarrow+\infty$ if and only if $c_{1}=c_{2}=0$ and $\left|c_{3}\right|+\left|c_{4}\right| \neq 0$, i.e., if and only if $\vec{u}$ has the form (4.3). Hence the theorem is proved in the case $|\omega|=0$.

Next, suppose $|\omega| \neq 0$. Since $M(\omega, s)$ in (4.4) is no longer a diagonal matrix, the ordinary differential system (4.4) is not a decoupled system as in the case $|\omega|=0$. However, following the derivation of the perfectly absorbing boundary conditions given in $\S 2.1$ (cf. (2.10)-(2.14)), we can show that the general solution of (4.4) is given by

$$
\vec{\varphi}\left(x_{1}\right)=\left(c_{1} \vec{a}_{1}+c_{2} \vec{b}_{1}\right) e^{\lambda_{1} x_{1}}+\left(c_{3} \vec{a}_{2}+c_{4} \vec{b}_{2}\right) e^{\lambda_{2} x_{1}},
$$

for all $\operatorname{Re} s \geq 0$. Here

$$
\begin{aligned}
& \vec{a}_{1}=\left(\frac{-i|\omega|}{\lambda_{1}}, \omega_{2}^{\prime}, \omega_{3}^{\prime}, 0, \frac{\omega_{3}^{\prime} s}{\lambda_{1}},-\frac{\omega_{2}^{\prime} s}{\lambda_{1}}\right)^{t}, \quad \vec{b}_{1}=\left(0, \frac{\omega_{3}^{\prime} s}{\lambda_{1}},-\frac{\omega_{2}^{\prime} s}{\lambda_{1}}, \frac{i|\omega|}{\lambda_{1}},-\omega_{2}^{\prime},-\omega_{3}^{\prime}\right)^{t}, \\
& \vec{a}_{2}=\left(\frac{-i|\omega|}{\lambda_{2}}, \omega_{2}^{\prime}, \omega_{3}^{\prime}, 0, \frac{\omega_{3}^{\prime} s}{\lambda_{2}},-\frac{\omega_{2}^{\prime} s}{\lambda_{2}}\right)^{t}, \quad \vec{b}_{2}=\left(0, \frac{\omega_{3}^{\prime} s}{\lambda_{2}},-\frac{\omega_{2}^{\prime} s}{\lambda_{2}}, \frac{i|\omega|}{\lambda_{2}},-\omega_{2}^{\prime},-\omega_{3}^{\prime}\right)^{t},
\end{aligned}
$$

and $c_{1}, \cdots, c_{4}$ are arbitrary complex constants. Hence the general solution of the form (4.1) of the equation (4.2) is again given by (4.6) with the above $\vec{a}_{j}$ and $\vec{b}_{j}$.

Using the same argument as in the case $|\omega|=0$, we conclude that (nonzero) $\vec{u} \rightarrow 0$ as $x_{1} \rightarrow+\infty$ if and only if $c_{1}=c_{2}=0$ and $\left|c_{3}\right|+\left|c_{4}\right| \neq 0$, i.e., if and only if $\vec{u}$ has the form (4.3). So the theorem holds in the case $|\omega| \neq 0$. The proof now is completed.

Remark 4.2. As a direct byproduct of the above proof, we have shown that the general solution of the associated homogeneous equation of the transformed Maxwell system (2.9) is given by

$$
\overrightarrow{\hat{u}}\left(x_{1}\right)=\left(c_{1} \vec{a}_{1}+c_{2} \vec{b}_{1}\right) e^{\lambda_{1} x_{1}}+\left(c_{3} \vec{a}_{2}+c_{4} \vec{b}_{2}\right) e^{\lambda_{2} x_{1}},
$$


for arbitrary complex constants $c_{1}, \cdots, c_{4}$, and the vectors $\vec{a}_{j}$ and $\vec{b}_{j}$ are defined by (4.5) when $|\omega|=0$ and by (4.7) when $|\omega| \neq 0$.

Another byproduct of the proof of Theorem 4.1, this one indirect, is that every $L^{2}([0,+\infty))$-integrable (in $\left.x_{1}\right)$ solution of the corresponding homogeneous equation of (2.9) must have the following form:

$$
\overrightarrow{\hat{u}}\left(x_{1}\right)=\left(c_{3} \vec{a}_{2}+c_{4} \vec{b}_{2}\right) e^{\lambda_{2} x_{1}} .
$$

We now are ready to state our third main theorem of this paper.

Theorem 4.2. The boundary condition defined by (2.19) satisfies the Kreiss condition. Each of the boundary conditions defined by (2.20), (2.21), (2.26) and (2.27) satisfies the Kreiss condition, except for the case $|\omega|=0$.

Proof. We only give the proof for boundary conditions (2.19), (2.20) and (2.21), since the proofs for boundary conditions (2.26) and (2.27) are same as those for (2.20) and (2.21).

First, we consider the case $|\omega| \neq 0$. From the Kreiss condition and Theorem 4.1 we know that it suffices to show that any solution of (4.2) of the form (4.3) does not satisfy the boundary conditions for $\operatorname{Re} s \geq 0$. Notice that $E_{1}=u_{1}, E_{2}=u_{2}$, $E_{3}=u_{3} ; H_{1}=u_{4}, H_{2}=u_{5}$, and $H_{3}=u_{6}$, where $u_{j}$ is the $j$ th component of the vector function $\vec{u}$ in (4.3). Let $\ell(x)=i \omega_{2} x_{2}+i \omega_{3} x_{3}$; from (4.3) we know that when $|\omega| \neq 0$ the decaying solutions of (4.2) of the form (4.3) are given by

$$
\begin{aligned}
& E_{1}=\frac{c_{3}|\omega| i}{\sqrt{|\omega|^{2}+s^{2}}} e^{\lambda_{2} x_{1}+\ell(x)+s t}, \\
& E_{2}=\left(c_{3} \omega_{2}^{\prime}-\frac{c_{4} \omega_{3}^{\prime} s}{\sqrt{|\omega|^{2}+s^{2}}}\right) e^{\lambda_{2} x_{1}+\ell(x)+s t}, \\
& E_{3}=\left(c_{3} \omega_{3}^{\prime}+\frac{c_{4} \omega_{2}^{\prime} s}{\sqrt{|\omega|^{2}+s^{2}}}\right) e^{\lambda_{2} x_{1}+\ell(x)+s t}, \\
& H_{1}=\frac{-c_{4}|\omega| i}{\sqrt{|\omega|^{2}+s^{2}}} e^{\lambda_{2} x_{1}+\ell(x)+s t} \\
& H_{2}=\left(\frac{-c_{3} \omega_{3}^{\prime} s}{\sqrt{|\omega|^{2}+s^{2}}}-c_{4} \omega_{2}^{\prime}\right) e^{\lambda_{2} x_{1}+\ell(x)+s t}, \\
& H_{3}=\left(\frac{c_{3} \omega_{2}^{\prime} s}{\sqrt{|\omega|^{2}+s^{2}}}-c_{4} \omega_{3}^{\prime}\right) e^{\lambda_{2} x_{1}+\ell(x)+s t} .
\end{aligned}
$$

By direct computations we get that for any $x \in \Gamma$

$$
\begin{aligned}
& \left(\begin{array}{l}
E_{2}+H_{3} \\
E_{3}-H_{2}
\end{array}\right)=k_{0}\left(\begin{array}{cc}
\omega_{2}^{\prime} & -\omega_{3}^{\prime} \\
\omega_{3}^{\prime} & \omega_{2}^{\prime}
\end{array}\right)\left(\begin{array}{l}
c_{3} \\
c_{4}
\end{array}\right), \\
& \left(\begin{array}{l}
\frac{\partial E_{1}}{\partial t}+\frac{\partial E_{2}}{\partial x_{2}}+\frac{\partial E_{3}}{\partial x_{3}} \\
\frac{\partial H_{1}}{\partial t}+\frac{\partial H_{2}}{\partial x_{2}}+\frac{\partial H_{3}}{\partial x_{3}}
\end{array}\right)=k_{1}\left(\begin{array}{cc}
1 & 0 \\
0 & -1
\end{array}\right)\left(\begin{array}{l}
c_{3} \\
c_{4}
\end{array}\right)
\end{aligned}
$$

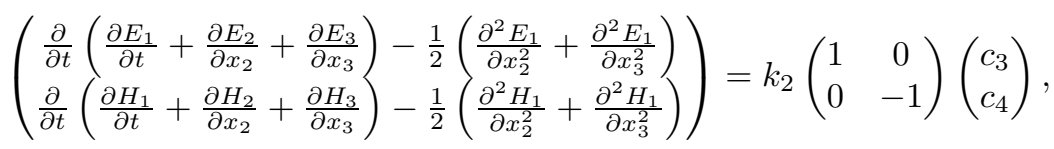


where

$$
\begin{gathered}
k_{0}=\left(1+\frac{s}{\sqrt{|\omega|^{2}+s^{2}}}\right) e^{\ell(x)+s t}, \quad k_{1}=i\left(1+\frac{|\omega| s}{\sqrt{|\omega|^{2}+s^{2}}}\right) e^{\ell(x)+s t} \\
k_{2}=i\left(s+\frac{|\omega|\left(2 s^{2}+|\omega|^{2}\right)}{2 \sqrt{|\omega|^{2}+s^{2}}}\right) e^{\ell(x)+s t}
\end{gathered}
$$

Since $|\omega| \neq 0$, clearly, $k_{j} \neq 0, j=0,1,2$, for $\operatorname{Re} s \geq 0$. Hence each right hand side of (4.8)-(4.10) is a zero vector if and only if $c_{3}=c_{4}=0$. That is, there is no nonzero solution of (4.2) of the form (4.3) which satisfies the boundary conditions (2.19), (2.20) and (2.21) in the case $|\omega| \neq 0$.

Next, we consider the case $|\omega|=0$. From (4.3) we know that when $|\omega|=0$ the decaying solutions of (4.2) of the form (4.3) are given by

$$
\begin{array}{ll}
E_{1} \equiv 0, & H_{1} \equiv 0, \\
E_{2}=c_{3} e^{-s\left(x_{1}-t\right)}, & H_{2}=-c_{4} e^{-s\left(x_{1}-t\right)}, \\
E_{3}=c_{4} e^{-s\left(x_{1}-t\right)}, & H_{3}=c_{3} e^{-s\left(x_{1}-t\right)} .
\end{array}
$$

Recall that $s$ must be nonzero, as otherwise there is no decaying solution of (4.2) of the form (4.3) when $|\omega|=0$.

Since $E_{1} \equiv H_{1} \equiv 0$ and $E_{2}, E_{3}, H_{2}, H_{3}$ all are independent of $x_{2}$ and $x_{3}$, it is easy to check that the above solutions do not satisfy the boundary condition (2.19) unless $c_{3}=c_{4}=0$. However, they do satisfy the boundary conditions (2.20) and (2.21) for any complex constants $c_{3}$ and $c_{4}$. The proof is now completed.

4.2. Remarks on the case $|\omega|=0$. In the previous subsection we found that for the first order and second order boundary conditions (2.20), (2.21), (2.26) and (2.27) there is a breakdown in the Kreiss condition when $|\omega|=0$. It is known that breakdowns in the Kreiss condition also occur for absorbing boundary conditions for acoustic and elastic waves, but only in the case of zero frequency and zero wave numbers (cf. [9], [11] and references therein); that is, it happens when $s=|\omega|=0$. So the situation for the electromagnetic wave equations with the proposed absorbing boundary conditions is a bit different from those for acoustic and elastic waves.

The purpose of this subsection is to briefly point out three cures for the stability breakdown seen in the previous subsection for electromagnetic waves at angle of incidence zero. For the sake of definiteness and simplicity, we only use the boundary condition (2.20) as an example to explain the ideas, which can be applied easily to the boundary conditions (2.21), (2.26) and (2.27).

The first cure is a well-known one (cf. [9], [11]): to remove the instability by removing the incompatibility between the initial data and the boundary condition. This can be achieved by doing a suitable initialization of the boundary condition. For more discussion in this direction, we refer to [10].

To introduce the second cure for the instability, we recall that to derive (2.20) we first approximate $\sqrt{1-|\omega|^{2} / \xi^{2}}$ by 1 in (2.18), to get

$$
\begin{array}{ll}
\left(\omega_{2} \hat{E}_{2}+\omega_{3} \hat{E}_{3}\right)-\left(\omega_{3} \hat{H}_{2}-\omega_{2} \hat{H}_{3}\right)=0, & \text { on } \Gamma, \\
\left(\omega_{2} \hat{H}_{2}+\omega_{3} \hat{H}_{3}\right)+\left(\omega_{3} \hat{E}_{2}-\omega_{2} \hat{E}_{3}\right)=0, & \text { on } \Gamma,
\end{array}
$$

and then apply the inverse Fourier transform. However, since the inverse Fourier transform is uniquely determined up to an additive null function (zero function 
class in $\left.L^{2}\right)$, more precisely, let $x^{\prime}=\left(x_{1}, x_{2}\right)$, from (4.12) we should get

$$
\begin{aligned}
& \frac{\partial E_{2}}{\partial x_{2}}+\frac{\partial E_{3}}{\partial x_{3}}-\left(\frac{\partial H_{2}}{\partial x_{3}}-\frac{\partial H_{3}}{\partial x_{2}}\right)=m\left(x^{\prime}, t\right), \quad \text { on } \Gamma, \\
& \left(\frac{\partial E_{2}}{\partial x_{3}}-\frac{\partial E_{3}}{\partial x_{2}}\right)+\frac{\partial H_{2}}{\partial x_{2}}+\frac{\partial H_{3}}{\partial x_{3}}=n\left(x^{\prime}, t\right), \quad \text { on } \Gamma,
\end{aligned}
$$

for some $m\left(x^{\prime}, t\right)$ and $n\left(x^{\prime}, t\right)$ such that

$$
\int\left|m\left(x^{\prime}, t\right)\right|^{2} d x^{\prime} d t=0, \quad \int\left|n\left(x^{\prime}, t\right)\right|^{2} d x^{\prime} d t=0 .
$$

Therefore, (2.20) becomes

$$
\begin{array}{ll}
\frac{\partial E_{1}}{\partial t}+\frac{\partial E_{2}}{\partial x_{2}}+\frac{\partial E_{3}}{\partial x_{3}}=m\left(x^{\prime}, t\right), & \text { on } \Gamma, \\
\frac{\partial H_{1}}{\partial t}+\frac{\partial H_{2}}{\partial x_{2}}+\frac{\partial H_{3}}{\partial x_{3}}=n\left(x^{\prime}, t\right), & \text { on } \Gamma .
\end{array}
$$

It is not hard to check that if one of $m\left(x^{\prime}, t\right)$ and $n\left(x^{\prime}, t\right)$ is not identically equal to zero, say, $m\left(x^{\prime}, t\right)=0.1$ for $x_{2}=x_{3}=0$ and $m\left(x^{\prime}, t\right)=0$ otherwise, then the boundary condition (4.14) satisfies the Kreiss condition. It is interesting to note that if one chooses $m\left(x^{\prime}, t\right) \equiv \epsilon_{1}$ and $n\left(x^{\prime}, t\right) \equiv \epsilon_{2}$, this cure is similar to the one proposed by Higdon in [9] and [11] for the acoustic and elastic wave equations. Since this stabilization approach will cause some additional reflections, the constants $\epsilon_{1}$ and $\epsilon_{2}$ must be chosen with care. For more discussion in this direction, we refer to [9] and [11].

The last cure for the instability is a variant of the previous one. Since the Fourier transform is defined uniquely up to an additive null function, we can divide both sides of (4.12.i) and (4.12.ii) by $\omega_{2}$ to get

$$
\begin{aligned}
& \hat{E}_{2}+\hat{H}_{3}-\frac{\omega_{3}}{\omega_{2}}\left(\hat{H}_{2}-\hat{E}_{3}\right)=0, \\
& \hat{H}_{2}-\hat{E}_{3}+\frac{\omega_{3}}{\omega_{2}}\left(\hat{E}_{2}+\hat{H}_{3}\right)=0,
\end{aligned}
$$

Applying the inverse Fourier transform, we obtain

$$
\begin{aligned}
& E_{2}+H_{3}-\int_{0}^{x_{2}}\left(\frac{\partial H_{2}}{\partial x_{3}}-\frac{\partial E_{2}}{\partial x_{3}}\right) d x_{2}^{\prime}=m\left(x^{\prime}, t\right), \quad \text { on } \Gamma, \\
& H_{2}-E_{3}+\int_{0}^{x_{2}}\left(\frac{\partial E_{2}}{\partial x_{3}}+\frac{\partial H_{3}}{\partial x_{3}}\right) d x_{2}^{\prime}=n\left(x^{\prime}, t\right), \quad \text { on } \Gamma .
\end{aligned}
$$

where $m\left(x^{\prime}, t\right)$ and $n\left(x^{\prime}, t\right)$ are two null functions in $L^{2}$. One can show that the boundary condition (4.16) satisfies the Kreiss condition for any null functions $m\left(x^{\prime}, t\right)$ and $n\left(x^{\prime}, t\right)$. On the other hand, since (4.16) involves integrations in $x_{2}$, it is a "weakly" global boundary condition.

\section{§5. NumERICAL EXPERIMENTS}

In this section we present some numerical experiments to show the effectiveness of the absorbing boundary conditions developed in this paper. For simplicity all computations are done in two space dimensions.

To get the two space dimensional Maxwell system, one assumes that all the field and source quantities are independent of one spatial variable, say $x_{3}$. Then it is 
easy to check that the Maxwell system (2.1) with zero source is reduced to the following two decoupled systems of three equations:

$$
\begin{aligned}
& \frac{\partial E_{1}}{\partial t}=\frac{1}{\epsilon} \frac{\partial H_{3}}{\partial x_{2}} \\
& \frac{\partial E_{2}}{\partial t}=-\frac{1}{\epsilon} \frac{\partial H_{3}}{\partial x_{1}} \\
& \frac{\partial H_{3}}{\partial t}=\frac{1}{\mu}\left(\frac{\partial E_{1}}{\partial x_{2}}-\frac{\partial E_{2}}{\partial x_{1}}\right) ;
\end{aligned}
$$

and

$$
\begin{aligned}
\frac{\partial H_{1}}{\partial t} & =-\frac{1}{\mu} \frac{\partial E_{3}}{\partial x_{2}} \\
\frac{\partial H_{2}}{\partial t} & =\frac{1}{\mu} \frac{\partial E_{3}}{\partial x_{1}}, \\
\frac{\partial E_{3}}{\partial t} & =\frac{1}{\epsilon}\left(\frac{\partial H_{2}}{\partial x_{1}}-\frac{\partial H_{1}}{\partial x_{2}}\right) .
\end{aligned}
$$

The solutions of systems (5.1) and (5.2) are often referred as Transverse Electric (TE) waves and Transverse Magnetic (TM) waves, respectively, in the literature. In this section numerical tests are done only for TE waves, but all results are also valid for TM waves because of the symmetry in $\vec{E}$ and $\vec{H}$ of the absorbing boundary conditions.

The finite difference method is used to discretize both the interior equations (5.1) and the boundary conditions (2.20) and (2.21). Specifically, Yee's second order leapfrog finite difference scheme is adopted to discretize the interior equations (cf. [22], also see [18]), and Mur's idea is used to discretize the boundary conditions (2.20) and (2.21) so that the truncation errors on the boundary have the same order as Yee's method (cf. [16], [18]).

All computations described here are done on square grids, that is, $h=\Delta x_{1}=$ $\Delta x_{2}$, and the size of $h$ is chosen according to the size of the frequency of the test problem so that there are about 15 grid points per wave length. Since Yee's scheme is an explicit scheme, the time step $\Delta t$ must be chosen to satisfy the CFL (Courant-Friedrichs-Levy) condition for the stability. It is well known that the CFL condition in two space dimensions for Yee's scheme is given by (cf. [22])

$$
\Delta t \leq \frac{h}{c \sqrt{2}}, \quad c=\frac{1}{\sqrt{\epsilon \mu}},
$$

where $c$ is the speed of the electromagnetic wave. In this section our test problem is formulated in terms of nondimensional variables for which $c=1$.

The sizes of the reflections are measured using discrete $L^{2}$ and $L^{\infty}$ norms of the error functions over the computational domain. The error functions are the differences between the free space (numerical) true solutions, which are known in all tests, and the computed solutions with the absorbing boundary conditions.

Test problem. We consider the Maxwell system (5.1) on the square domain $\Omega=$ $[0,1]^{2}$, which has sinusoidal solutions of the form

$$
\begin{aligned}
& E_{1}=a_{1} \sin \left(\omega t+\alpha_{1} x_{1}+\alpha_{2} x_{2}\right), \\
& E_{2}=a_{2} \sin \left(\omega t+\alpha_{1} x_{1}+\alpha_{2} x_{2}\right), \\
& H_{3}=a_{3} \sin \left(\omega t+\alpha_{1} x_{1}+\alpha_{2} x_{2}\right),
\end{aligned}
$$


where

$$
a_{1} \omega=a_{3} \alpha_{2}, \quad a_{2} \omega=-a_{3} \alpha_{1}, \quad a_{3} \omega=a_{1} \alpha_{2}-a_{2} \alpha_{1} .
$$

It is easy to check that (5.4.iv) implies the dispersion relation

$$
\omega^{2}=\alpha_{1}^{2}+\alpha_{2}^{2} \text {. }
$$

Note that we have assumed that $\epsilon=\mu=1$. Since the absorbing boundary conditions of this paper are developed for the half-space domain, in the tests described below we only consider the case that one edge $\Gamma_{1}=\left\{\left(0, x_{2}\right) ; 0<x_{2}<1\right\}$ of $\Omega$ is the artificial boundary, and the true values of the exact solution are used as boundary conditions on the other parts of the boundary.

Below, we present the computational results of two tests for the example discussed above (after 30 time steps). In the first test we choose the frequency $\omega=15$ and $h=1 / 40$, while in the second test we have $\omega=30$ and $h=1 / 80$. But in both tests we take $a_{3}=2.5$ and $\Delta t=h / 5$. Tables 1 and 3 contain the results obtained by using the first order absorbing boundary condition (2.20), and Tables 2 and 4 contain the results obtained by using the second order absorbing boundary condition (2.21). The graphs of the computed $E_{1}$ field and its reflection caused by the absorbing boundary conditions (2.20) and (2.21) when $\omega=15, \theta=14.8^{\circ}$ are shown in Figures of Test 1; the graphs of the computed $H_{3}$ field and its reflection when $\omega=30, \theta=29.9^{\circ}$ are given in Figures of Test 2. As expected, the second order boundary condition (2.21) performs much better than the first order boundary condition (2.20) does. One reason for that, which can be seen from the computation results, is that the reflection produced by using (2.20) propagates back into the computational domain at a faster rate than the reflection produced using (2.21). After 30 time iterations, the former propagates about 5 grids into the computational domain, while the later moves about 3 grids into the computational domain. Finally, it is also worth mentioning that the computation results indicate that the reflections using two boundary conditions are independent of the frequency.

TABLE 1. The relative reflections produced by (2.20) with $\omega=15$

\begin{tabular}{|c|l|l|l|l|l|l|l|l|}
\hline angle & \multicolumn{2}{|c|}{$\theta=2.1^{\circ}$} & \multicolumn{2}{c|}{$\theta=14.8^{\circ}$} & \multicolumn{2}{c|}{$\theta=29.9^{\circ}$} & \multicolumn{2}{c|}{$\theta=36.9^{\circ}$} \\
\hline reflection (\%) & $L^{2}$ & $L^{\infty}$ & $L^{2}$ & $L^{\infty}$ & $L^{2}$ & $L^{\infty}$ & $L^{2}$ & $L^{\infty}$ \\
\hline \hline$E_{1}$ & 0.608 & 2.319 & 0.925 & 3.933 & 2.589 & 10.965 & 3.731 & 15.576 \\
\hline$E_{2}$ & 0.413 & 1.467 & 0.884 & 2.767 & 2.821 & 9.227 & 4.469 & 15.222 \\
\hline$H_{3}$ & 0.380 & 1.154 & 0.804 & 2.482 & 2.245 & 7.620 & 3.189 & 11.308 \\
\hline
\end{tabular}

TABLE 2. The relative reflections produced by (2.21) with $\omega=15$

\begin{tabular}{|c|l|l|l|l|l|l|l|l|}
\hline angle & \multicolumn{2}{|c|}{$\theta=2.1^{\circ}$} & \multicolumn{2}{c|}{$\theta=14.8^{\circ}$} & \multicolumn{2}{c|}{$\theta=29.9^{\circ}$} & \multicolumn{2}{c|}{$\theta=36.9^{\circ}$} \\
\hline reflection (\%) & $L^{2}$ & $L^{\infty}$ & $L^{2}$ & $L^{\infty}$ & $L^{2}$ & $L^{\infty}$ & $L^{2}$ & $L^{\infty}$ \\
\hline \hline$E_{1}$ & 0.048 & 0.965 & 0.080 & 0.479 & 0.130 & 0.727 & 0.117 & 0.664 \\
\hline$E_{2}$ & 0.261 & 1.016 & 0.224 & 1.038 & 0.305 & 1.539 & 0.312 & 1.692 \\
\hline$H_{3}$ & 0.223 & 0.851 & 0.182 & 0.857 & 0.219 & 1.121 & 0.205 & 1.165 \\
\hline
\end{tabular}



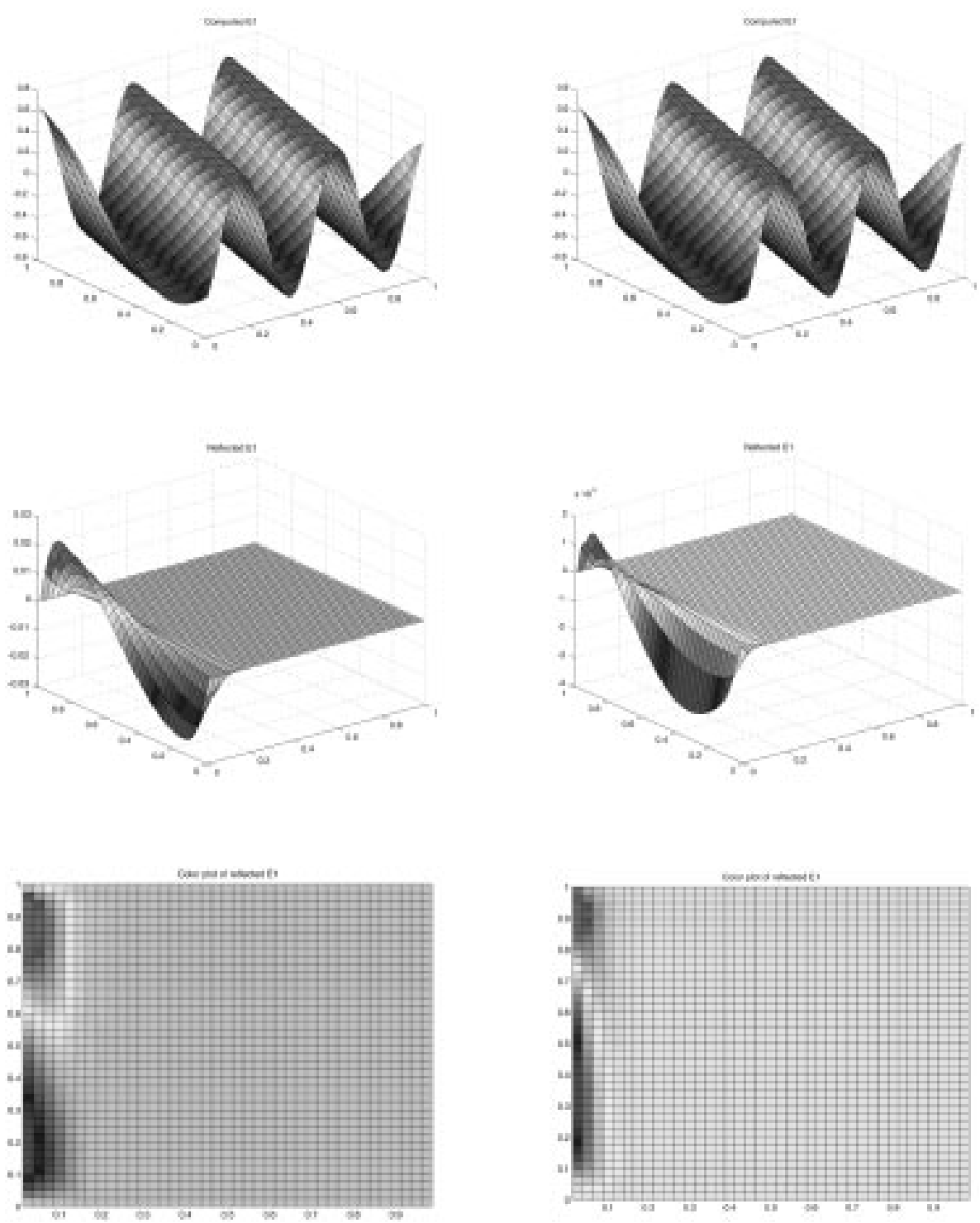

Figures of Test 1. ABC (2.20) is used to produce the left column and $\mathrm{ABC}$ (2.21) is used to produce the right column with $\omega=15, \theta=14.8^{\circ}$ 

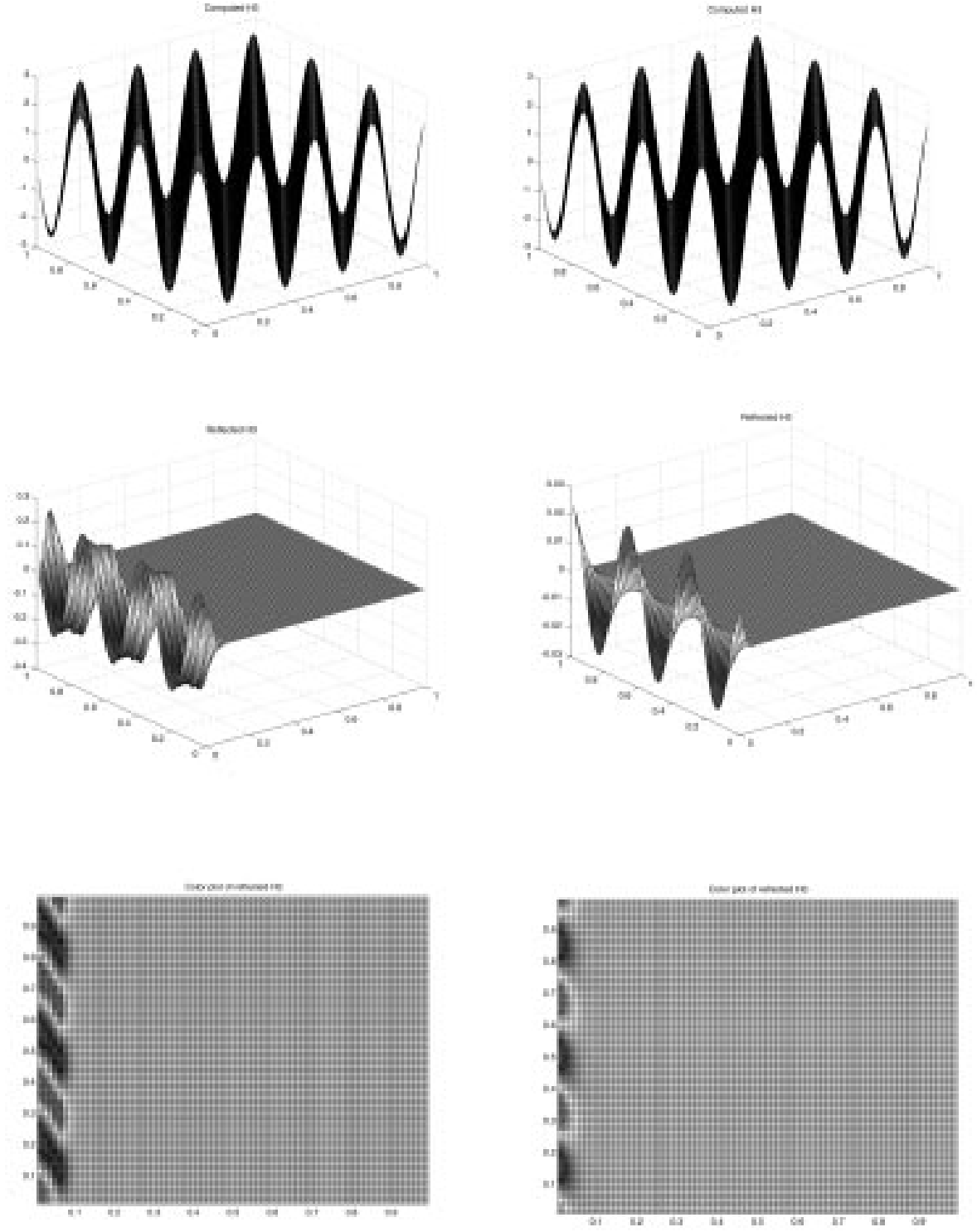

Figures of Test 2. ABC (2.20) is used to produce the left column and $\mathrm{ABC}$ (2.21) is used to produce the right column with $\omega=30, \theta=29.9^{\circ}$ 
TABLE 3 . The relative reflections produced by (2.20) with $\omega=30$

\begin{tabular}{|c|l|l|l|l|l|l|l|l|}
\hline angle & \multicolumn{2}{|c|}{$\theta=2.1^{\circ}$} & \multicolumn{2}{c|}{$\theta=14.8^{\circ}$} & \multicolumn{2}{c|}{$\theta=29.9^{\circ}$} & \multicolumn{2}{c|}{$\theta=36.9^{\circ}$} \\
\hline reflection $(\%)$ & $L^{2}$ & $L^{\infty}$ & $L^{2}$ & $L^{\infty}$ & $L^{2}$ & $L^{\infty}$ & $L^{2}$ & $L^{\infty}$ \\
\hline \hline$E_{1}$ & 0.356 & 2.307 & 0.684 & 3.950 & 1.911 & 10.980 & 2.722 & 15.653 \\
\hline$E_{2}$ & 0.328 & 1.457 & 0.622 & 2.766 & 2.018 & 9.219 & 3.163 & 15.220 \\
\hline$H_{3}$ & 0.317 & 1.353 & 0.562 & 2.483 & 1.587 & 7.167 & 2.243 & 9.869 \\
\hline
\end{tabular}

TABLE 4. The relative reflections produced by (2.21) with $\omega=30$

\begin{tabular}{|c|l|l|l|l|l|l|l|l|}
\hline angle & \multicolumn{2}{|c|}{$\theta=2.1^{\circ}$} & \multicolumn{2}{c|}{$\theta=14.8^{\circ}$} & \multicolumn{2}{c|}{$\theta=29.9^{\circ}$} & \multicolumn{2}{c|}{$\theta=36.9^{\circ}$} \\
\hline reflection (\%) & $L^{2}$ & $L^{\infty}$ & $L^{2}$ & $L^{\infty}$ & $L^{2}$ & $L^{\infty}$ & $L^{2}$ & $L^{\infty}$ \\
\hline \hline$E_{1}$ & 0.040 & 0.961 & 0.061 & 0.479 & 0.094 & 0.729 & 1.108 & 0.818 \\
\hline$E_{2}$ & 0.186 & 1.012 & 0.152 & 1.037 & 0.221 & 1.538 & 0.252 & 1.711 \\
\hline$H_{3}$ & 0.152 & 0.848 & 0.122 & 0.858 & 0.159 & 1.121 & 0.164 & 1.124 \\
\hline
\end{tabular}

\section{ACKNOWLEDGMENTS}

I would like to thank Professor Jim Douglas, Jr. for suggesting that I work on this problem. Thanks are also due to Professor Jean Roberts for her helpful discussions, in particular, for her help in getting several references. The last section of this paper was completed while I was visiting the IMA in Spring 1997, I would like to thank the IMA for its support and hospitality. Finally, I would also like to thank the referees for their valuable remarks and suggestions.

\section{REFERENCES}

1. A. Bendali and L. Halpern, Conditions aux limites absorbantes pour le système de Maxwell dans le vide en dimension 3, C.R.A.S. Paris, Tome 307, Série I, no 20 (1988), 1011-1013. MR 90f: 35172

2. B. Chalinder, Conditions aux limites absorbantes pour les équations de l' élastodynamique linéaire, Ph.D. Thesis, Université de Saint-Etienne, 1988.

3. R. Dautray and J. L. Lions, Mathematical Analysis and Numerical Methods for Science and Technology, I, Springer-Verlag, New York, 1990. MR 90k:00004

4. E. Duceau and B. Mercier, Approche instationnaire pour la réflexion des ondes électromagnétiques, Communication aux journées "Aspects Mathématiques des Phénomènes de Propagation d'ondes", INRIA, Nice 16 (1988).

5. B. Engquist and A. Majda, Absorbing boundary conditions for the numerical simulation of waves, Math. Comp. 31 (1977), 629-651. MR 55:9555

6. _ Radiation boundary conditions for acoustic and elastic wave calculations, Comm. Pure Appl. Math. 32 (1979), 313-357. MR 80e:76041

7. R. Hersh, Mixed problems in several variables, J. Math. Mech. 12 (1963), 317-334. MR 26:5304

8. R. L. Higdon, Initial-boundary value problems for linear hyperbolic systems, SIAM Rev. 28 (1986), 177-217. MR 88a:35138

9. —_ Absorbing boundary conditions for difference approximations to the multidimensional wave equation, Math. Comp. 47 (1986), 437-459. MR 87m:65131

10. _ Numerical absorbing boundary conditions for the wave equation, Math Comp 49 (1987), 65-90. MR 88f:65168 
11. _ Radiation boundary conditions for elastic wave propagation, SIAM J. Numer. Anal. 27 (1990), 831-870. MR 91h:73017

12. P. Joly and B. Mercier, Une nouvelle condition transparente d'order 2 pour les équations de Maxwell en dimension 3, Rapport INRIA, $\mathrm{n}^{0} 1047$ (1989).

13. H. O. Kreiss, Initial boundary value problems for hyperbolic systems, Comm. Pure Appl. Math. 23 (1970), 277-298. MR 55:10862

14. L. Landau and E. Lifschitz, The Classical Theory of Fields, Pergamon, Oxford, 1959. MR 13:289i

15. A. Majda and S. Osher, Initial-boundary value problems for hyperbolic equations with uniformly characteristic boundary, Comm. Pure Appl. Math. 28 (1975), 607-675. MR 53:13857

16. G. Mur, Absorbing boundary conditions for the finite-difference approximation of the timedomain electromagnetic-field equations, IEEE Trans. Electromag. Comp. EMC 23 (1981), 377.

17. M. Sesquès, Conditions aux limites absorbantes pour le système de Maxwell, Ph. D. thesis, Université de Bordeaux, 1990.

18. A. Taflove, Computational Electromagnetics, the Finite-Difference Time-Domain Method, Artech House, 1995.

19. H. F. Tiersten, A Development of the Equations of Electromagnetism in Material Continua, Springer Tracts in Natural Philosophy Volume 36, Springer-Verlag, New York, 1990. MR 91e:78012

20. L. N. Trefethen, Instability of difference models for hyperbolic initial boundary value problems, Comm. Pure Appl. Math. 37 (1984), 329-367. MR 86f:65162

21. L. N. Trefethen and L. Halpern, Well-posedness of one-way wave equations and absorbing boundary conditions, Math. Comp. 47 (1986), 421-435. MR 88b:65148

22. K. S. Yee, Numerical solution of initial boundary value problems involving Maxwell's equations in isotropic media, IEEE Trans. Ant. Prop. AP-14 (1966), 302-307.

Department of Mathematics, The University of Tennessee, Knoxville, TN 37996

E-mail address: xfeng@math.utk.edu 